\section{The Path from $\beta$-Carotene to Carlactone, a Strigolactone-Like Plant Hormone}

Adrian Alder, ${ }^{1}$ Muhammad Jamil, ${ }^{2}$ Mattia Marzorati, ${ }^{3}$ Mark Bruno, ${ }^{1}$ Martina Vermathen, ${ }^{3}$ Peter Bigler, ${ }^{3}$ Sandro Ghisla, ${ }^{4}$ Harro Bouwmeester, ${ }^{2,5}$ Peter Beyer, ${ }^{1,6}$ Salim Al-Babilit, ${ }^{1,6 *}$

Strigolactones, phytohormones with diverse signaling activities, have a common structure consisting of two lactones connected by an enol-ether bridge. Strigolactones derive from carotenoids via a pathway involving the carotenoid cleavage dioxygenases 7 and 8 (CCD7 and CCD8) and the iron-binding protein D27. We show that D27 is a $\beta$-carotene isomerase that converts all-trans- $\beta$-carotene into 9-cis- $\beta$-carotene, which is cleaved by $C C D 7$ into a 9-cis-configured aldehyde. CCD8 incorporates three oxygens into 9-cis- $\beta$-apo-10'-carotenal and performs molecular rearrangement, linking carotenoids with strigolactones and producing carlactone, a compound with strigolactone-like biological activities. Knowledge of the structure of carlactone will be crucial for understanding the biology of strigolactones and may have applications in combating parasitic weeds.

$\mathrm{T}$ he biosynthetic carotenoid pathway pro vides precursors for smaller, physiologi cally important compounds such as the plant hormone abscisic acid (I), the verte brate morphogen retinoic acid (2), and the fungal pheromone trisporic acid (3), which originate from oxidative cleavage of $\mathrm{C} \mathrm{C}$ double bonds in carotenoid backbones (4 7). The derivative compounds, named apocarotenoids (see fig. S1 for nomenclature and structures), may undergo structural modifications concealing their origin. Strigolactones, a prominent example of such com pounds, are a group of metabolites with a com mon $\mathrm{C}_{19}$ structure (e.g., 5 deoxystrigol, Fig. 1A) consisting of a tricyclic lactone (A, B, and C rings) connected via an enol ether bridge to a second lactone (D ring) (8). Several lines of evidence dem onstrate that strigolactones derive from carotenoids. A putative biosynthetic pathway has been pro posed, leading from $\mathrm{C}_{15}$ apocarotenal to the $\mathrm{ABC}$ $\mathrm{C}_{14}$ skeleton, which is then coupled to a butenolide group (D ring) of unknown origin (9).

Released by plant roots, strigolactones trig ger seed germination of root parasitic weeds such as witchweeds (Striga spp.) (10) and can induce hyphal branching in arbuscular mycorrhizal fungi, an essential step in establishing the symbiosis

${ }^{1}$ Faculty of Biology, University of Freiburg, 79104 Freiburg, Germany. 'Laboratory of Plant Physiology, Wageningen Uni versity, 6700 AR Wageningen, Netherlands. ${ }^{3}$ Department of Chemistry and Biochemistry, University of Bern, 3012 Bern, Switzerland. ${ }^{4}$ Department of Biology, University of Konstanz, 78457 Konstanz, Germany. ${ }^{5}$ Centre for Biosystems Genomics, 6700 AR Wageningen, Netherlands. ${ }^{6}$ Centre for Biological Signalling Studies (Bioss), 79104 Freiburg, Germany.

*To whom correspondence should be addressed. E mail: salim.albabili@biologie.uni freiburg.de
(11). In plants, strigolactones inhibit tillering and shoot branching. High tillering (more branch ing) is observed in plant mutants disrupted in Carotenoid Cleavage Dioxygenase 7 (CCD7) and $C C D 8$, in Dwarf 27 (D27) encoding an iron binding polypeptide with unknown catalytic properties, or in a cytochrome P450 gene rep resented by More Axillary Growth 1 (MAXI) from

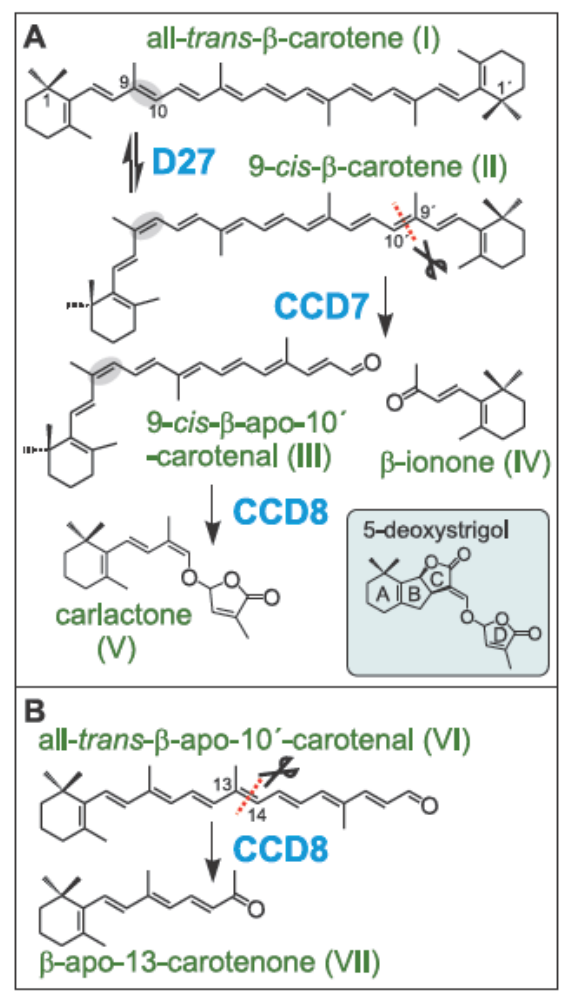

Arabidopsis thaliana $(8,12$ 17). The application of the synthetic strigolactone GR24 restored the wild type tillering/branching phenotype of these mutants, suggesting the involvement of $\mathrm{CCD} 7, \mathrm{CCD} 8, \mathrm{D} 27$, and MAX1 in strigolactone biosynthesis $(8,1217)$.

CCDs are nonheme iron enzymes that cut $\mathrm{C} \mathrm{C}$ double bonds by incorporating a dioxygen, yielding carbonyl products (4 7). It was sup posed that CCD7 from Arabidopsis (AtCCD7) cleaves all trans $\beta$ carotene $\left(\mathrm{C}_{40}\right.$; Fig. $\left.1 \mathrm{~A}\right)$ into all trans $\beta$ apo $10^{\prime}$ carotenal $\left(\mathrm{C}_{27}\right)$ that is then further shortened by AtCCD8 to the $\mathrm{C}_{18}$ ketone $\beta$ apo 13 carotenone (Fig. 1B) (18), a biolog ically active compound affecting the growth of root hairs in plants (19). The sequential activity of the two cleavage enzymes was deduced from the coexpression of AtCCD7 and AtCCD8 in Escherichia coli strains accumulating all trans $\beta$ carotene (18) and confirmed by in vitro studies showing the formation of $\beta$ apo 13 carotenone from all trans $\beta$ apo $10^{\prime}$ carotenal by CCD8 en zymes from different plant species (20). How ever, the structure of $\beta$ apo 13 carotenone has little in common with that of strigolactones, and treatment of rice $c c d 8$ mutant with this compound did not restore the wild type tillering phenotype (fig. S2).

We used an in vitro approach to investigate the activities of CCD7 and CCD8 from Arabidopsis,

Fig. 1. The pathway to carlactone. (A) D27 catalyzes the isomerization of the C9-C10 double bond (shaded) in all-trans- $\beta$-carotene $\left(\mathrm{I} ; \mathrm{C}_{40}\right)$, leading to 9 -cis- $\beta$ carotene (II) that is cleaved by CCD7 at the $\mathrm{C}^{\circ}, \mathrm{C} 10^{\prime}$ position into 9-cis- $\beta$-apo-10'-carotenal $\left(C_{27} ;\right.$ III) and $\beta$-ionone $\left(C_{13} ;\right.$ IV). A single enzyme, $C C D 8$, converts III to carlactone (V) that already contains the $D$ ring and the enol ether bridge of strigolactones such as 5-deoxystrigol (inset). (B) CCD8 also catalyzes a known CCD reaction by converting all-trans $-\beta$-apo- 10 '-carotenal into $\beta$-apo-13-carotenone. However, this reaction is slower than that with the cis isomer by about a factor of 10. 


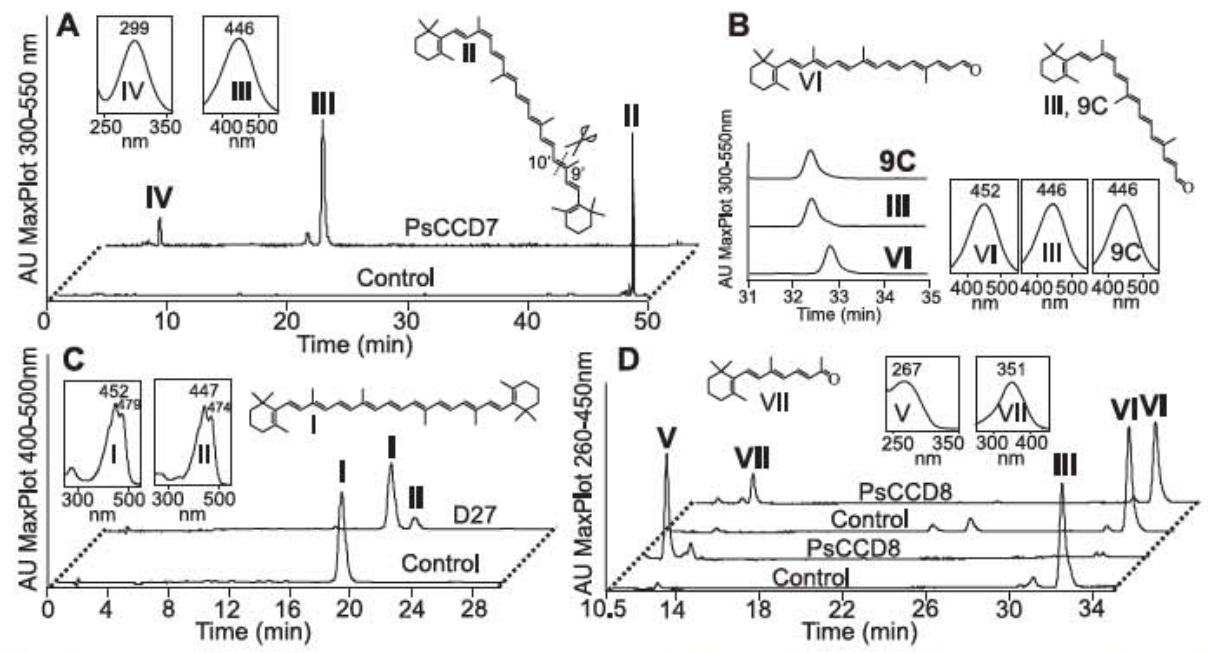

Fig. 2. HPLC analyses of in vitro incubations with PsCCD7, D27, and PsCCD8. (A) Incubation of PsCCD7

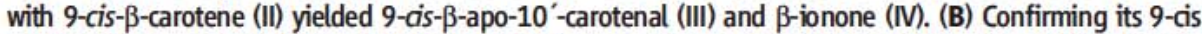
configuration, compound III coeluted with the reference compound 9-cis- $\beta$-apo-10'-carotenal (9C) 0.5 min earlier than with all-trans- $\beta$-apo-10'-carotenal (VI). (C) Incubation of purified D27 with all-trans- $\beta$ carotene (I) led to the formation of 9-cis- $\beta$-carotene (II). (D) PsCCD8 converted 9-cis- $\beta$-apo-10'carotenal (III) into an apocarotenoid (V) different from $\beta$-apo-13-carotenone (VII). The samples were analyzed using separation systems A (A), B [(B) and (D)], and C (C) (see supporting online material). UV-Vis spectra are depicted in the insets. AU, arbitrary units.

Table 1. ${ }^{1} \mathrm{H}$ and ${ }^{13} \mathrm{C}$ NMR data of compound V: experimental ${ }^{1} \mathrm{H}$ and ${ }^{13} \mathrm{C}$ chemical shifts, ${ }^{1} \mathrm{H}-{ }^{13} \mathrm{C}$ heteronuclear multiple bond correlations (HMBC), and relevant ${ }^{1} \mathrm{H}-{ }^{1} \mathrm{H}$ nuclear Overhauser enhancements (NOE) data for compound $\mathrm{V}$ dissolved in and referenced to $\mathrm{CD}_{2} \mathrm{Cl}_{2}\left({ }^{1} \mathrm{H}, 5.32 \mathrm{ppm} ;{ }^{13} \mathrm{C}, 54.0 \mathrm{ppm}\right)$.

\begin{tabular}{|c|c|c|c|c|}
\hline & ${ }^{1} \mathrm{H}$ & ${ }^{13} \mathrm{C}$ & ${ }^{1} \mathrm{H}={ }^{13} \mathrm{C} \mathrm{HMBC}$ & $1 D^{1} \mathrm{H}=\left\{{ }^{1} \mathrm{H}-\right\}=\mathrm{NOE}$ \\
\hline 1 & - & 34.37 & & \\
\hline 2 & $1.46(2 \mathrm{H}, \mathrm{m})$ & 39.86 & $\begin{array}{l}\mathrm{C}-1, \mathrm{C}-3, \mathrm{C}-4, \mathrm{C}-6, \mathrm{C}-16 \text { and C- } \\
17\end{array}$ & \\
\hline 3 & $1.61(2 \mathrm{H}, \mathrm{m})$ & 19.59 & $\mathrm{C}-1, \mathrm{C}-2, \mathrm{C}-4$ and $\mathrm{C}-5$ & \\
\hline 4 & $2.00(2 \mathrm{H}, \mathrm{brt}, 6.5 \mathrm{~Hz})$ & 33.24 & $\mathrm{C}-2, \mathrm{C}-3, \mathrm{C}-5, \mathrm{C}-6$ and $\mathrm{C}-18$ & \\
\hline 5 & - & 129.38 & & \\
\hline 6 & - & 138.18 & & \\
\hline 7 & $6.05(1 \mathrm{H}, \mathrm{d}, 16.2 \mathrm{~Hz})$ & 127.47 & C-5 and C-9 & $\mathrm{H}-16, \mathrm{H}-17$ and $\mathrm{H}-19$ \\
\hline 8 & $6.46(1 \mathrm{H}, \mathrm{d}, 16.4 \mathrm{~Hz})$ & 127.47 & C-6, C-9, C-10 and C-19 & no $\mathrm{NOE}$ for $\mathrm{H}=10$ \\
\hline 9 & - & 117.62 & & \\
\hline 10 & $6.20(1 \mathrm{H}, \mathrm{s})$ & 138.21 & $\begin{array}{l}\text { C-8, C-9, C-11 } \\
\text { and C-19 }\end{array}$ & $\begin{array}{l}\mathrm{H}-11 \text { and } \mathrm{H}-19 \\
\text { no } \mathrm{NOE} \text { for } \mathrm{H}-8\end{array}$ \\
\hline 11 & $5.97(1 \mathrm{H}, \mathrm{br} p, 1.5 \mathrm{~Hz})$ & 100.65 & C-10 & $\mathrm{H}-10$ and $\mathrm{H}-12$ \\
\hline 12 & $6.90(1 \mathrm{H}, \mathrm{br} \mathrm{p}, 1.5 \mathrm{~Hz})$ & 142,67 & & $\mathrm{H}-11$ and $\mathrm{H}-15$ \\
\hline 13 & - & 135.03 & & \\
\hline 14 & - & 171.52 & & \\
\hline 15 & $1.95(3 \mathrm{H}, \mathrm{t}, 1.5 \mathrm{~Hz})$ & 10.71 & $\begin{array}{l}\mathrm{C}-12, \mathrm{C}-13 \\
\text { and } \mathrm{C}-14\end{array}$ & $\mathrm{H}-12$ \\
\hline $\begin{array}{l}16, \\
17 \\
\end{array}$ & $1.00(6 \mathrm{H}, \mathrm{s})$ & 29.02 & $\begin{array}{l}\mathrm{C}-1, \mathrm{C}-2, \mathrm{C}-6, \mathrm{C}-7, \mathrm{C}-16 \text { and C- } \\
17\end{array}$ & \\
\hline 18 & $1.69(3 \mathrm{H}, \mathrm{s})$ & 21.69 & $\begin{array}{l}\text { C-4, C-5, C-6, C-7, C-16 and C- } \\
17\end{array}$ & \\
\hline 19 & $1.73(3 \mathrm{H}, \mathrm{d}, 1.2 \mathrm{~Hz})$ & 14.39 & $\begin{array}{l}\text { C-6, C-7, C-8 } \\
\text { and C-9 }\end{array}$ & \\
\hline
\end{tabular}

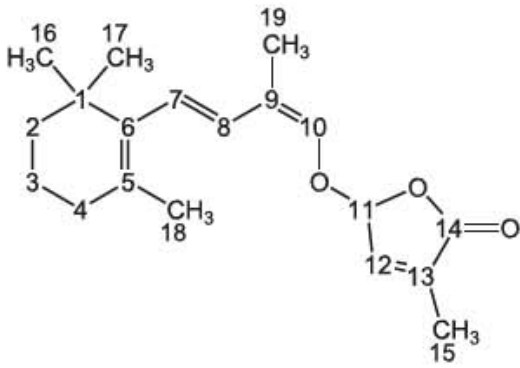

Pisum sativum, and Oryza sativa. Carotenoids occur in different cis configurations, a feature that may determine reaction specificities $(1,21)$. In deed, we show that the cis configuration of the $\mathrm{C} 9 \mathrm{C} 10$ double bond in $\beta$ carotene is decisive for the course of the reactions in strigolactone biosynthesis (Fig. 1) and that this pathway is char acterized by the function of a single enzyme, CCD8, in converting a 9 cis configured apocarotenal into a strigolactone like compound (Fig. 1A).

As shown by high performance liquid chro matography (HPLC) analysis, soluble fractions of $E$. coli cells expressing $P$. sativum $\mathrm{CCD} 7$ ( $\mathrm{PSCCD} 7)$ fused with thioredoxin (Fig. 2A), as well as puri fied $\mathrm{PsCCD} 7$ fused with glutathione $S$ transferase (GST; fig. S3), cleaved 9 cis $\beta$ carotene (II, $\mathrm{C}_{40}$ ) into 9 cis $\beta$ apo $10^{\prime}$ carotenal (III, $\mathrm{C}_{27}$ ) and $\beta$ ionone (IV, $\mathrm{C}_{13}$ ). All other $\beta$ carotene isomers tested, including all trans, 13 cis, and 15 cis $\beta$ carotene, were not converted (fig. S4, B to D). The products were identified by gas chromatog raphy and liquid chromatography coupled to mass spectrometry (GC MS and LC MS) (fig. S5) and according to their ultraviolet/visible (UV/Vis) absorption spectra. The configuration of $\beta$ apo $10^{\prime}$ carotenal (III) was confirmed by HPLC com parison (Fig. 2B) with the reference compounds all trans $\beta$ apo $10^{\prime}$ carotenal (VI) and 9 cis $\beta$ apo $10^{\prime}$ carotenal $(9 \mathrm{C})$. Arabidopsis and $O$. sativa thioredoxin $\mathrm{CCD} 7$ crude preparations exhibited the same activity (fig. S4A). Thus, CCD7 enzymes are stereospecific for the 9 cis configuration and cleave the $\mathrm{C}^{\prime} \mathrm{C}^{\prime} 0^{\prime}$ double bond in the trans moiety of 9 cis $\beta$ carotene (Fig. 1A).

In plants, the carotenoid biosynthetic path way provides $\beta$ carotene in the all trans configura tion (22), because the enzyme forming $\beta$ carotene, lycopene $\beta$ cyclase, acts as a selectivity filter for the all trans isomer (23). Cis isomers may arise nonspecifically from photo 60 isomerization (24) or thermo isomerization (25). Alternatively, a spe cific all trans $/ 9$ cis $\beta$ carotene isomerase may be postulated to divert the carotenoid pathway into strigolactone biosynthesis by forming the authentic CCD7 substrate. We tested whether D27, a strigolactone biosynthetic enzyme with hitherto unknown catalytic properties (26), exerts this function. In vitro incubation of all trans and 9 cis $\beta$ carotene with purified protein demon strates that D27 is indeed a carotene isomerase that reversibly converts all trans $\beta$ carotene into the $\mathrm{CCD} 7$ substrate 9 cis $\beta$ carotene (Fig. $2 \mathrm{C}$; see also fig. S6). These data suggest that D27 is the first all trans $/ 9$ cis $\beta$ carotene isomer ase reported so far and indicates that D27 is the enzyme providing the substrate for $\mathrm{CCD} 7$ in the course of strigolactone biosynthesis (Fig. 1A).

The configuration of the $\mathrm{C} 9 \mathrm{C} 10$ double bond of $\beta$ apo $10^{\prime}$ carotenal determines the na ture of the product formed by CCD8. Incubation of thioredoxin PsCCD8 crude preparations with the $\mathrm{CCD} 7$ product 9 cis $\beta$ apo $10^{\prime}$ carotenal yielded an unknown compound (V, Fig. 2D) with an absorption maximum at $267 \mathrm{~nm}$, lower than that of $\beta$ apo 13 carotenone (VII, Fig. 2D) reported 
to be formed by CCD8 from all trans $\beta$ apo $10^{\circ}$ carotenal $(19,20)$. Incubations of thioredoxin OsCCD8b and thioredoxin AtCCD8 led to the same results, demonstrating the generality of the finding that CCD8 enzymes use both $\beta$ apo $10^{\circ}$ carotenal isomers as substrates but can produce different products (Fig. 1 and fig. S7, A and B). Time courses of product formation (fig. S7C) show that the conversion of the cis substrate is faster than that of the trans substrate by a fac tor of $\sim 10$. We conclude that the conversion of the cis substrate into compound $\mathrm{V}$ is not a side reaction.

The structure of compound $\mathrm{V}$ was elucidated using electrospray ionization mass spectrom etry (ESI MS) and 1D and 2D nuclear mag netic resonance (NMR) spectroscopy, exploiting NMR reference data (27) and ${ }^{1} \mathrm{H},{ }^{13} \mathrm{C}$ chemical shift prediction (27). The NMR data are summa rized in Table 1. Both the ESI MS data ([M+H]': $303.19, \mathrm{C}_{19} \mathrm{H}_{26} \mathrm{O}_{3}$; fig. $\mathrm{S} 8$ ) and chemical shifts at $\mathrm{C} 9, \mathrm{C} 10$, and $\mathrm{C} 11$ pointed to oxygen as the linker between $\mathrm{C} 10$ and $\mathrm{C} 11$. This finding was

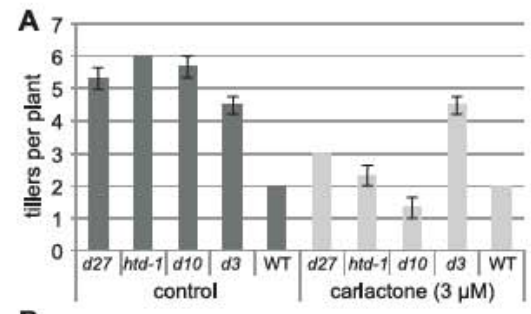

B

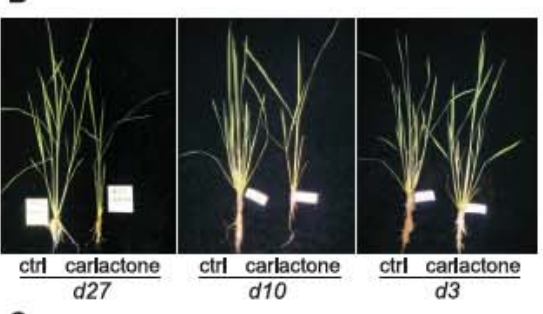

C

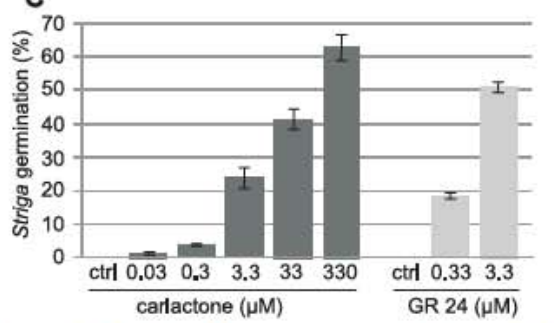

Fig. 3. Biological activity of carlactone. (A) Effect of carlactone on tillering in $d 27, h t d-1$, $d 10, d 3$, and the corresponding wild type (WT, Shiokari). Application of carlactone ( $3 \mu \mathrm{M}$ daily for 2 weeks) rescued the high-tillering phenotype of the strigolactone-deficient rice mutants, whereas $d 3$ and WT rice remained unaffected. (B) Photographs were taken after 30 days. (C) Germination rate of $S$. hermonthica seeds 2 days after treatment with different concentrations of carlactone, GR24, or water (ctrl). Data are means \pm SD [(A), $n=4 ;(C), n=4$ with 50 to 100 seeds per replicate]. corroborated by taking advantage of $2 \mathrm{D}{ }^{1} \mathrm{H}^{13} \mathrm{C}$ heteronuclear multiple bond correlation and $1 \mathrm{D}$ ${ }^{1} \mathrm{H}{ }^{1} \mathrm{H}$ nuclear Overhauser enhancement NMR spectroscopy (figs. S9 to S15). ${ }^{1} \mathrm{H}$ and ${ }^{13} \mathrm{C}$ chem ical shift prediction (28) confirmed the pro posed structure. Thus, compound V [IUPAC nomenclature: $2^{\prime}, 3^{\prime} Z, 4^{\prime} 5^{\prime} E 3$ methyl 5 (3' methyl $1^{\prime}$ oxa penta $2^{\prime}, 4^{\prime}$ dienyl $5^{\prime}\left(2^{\prime \prime}, 6^{\prime \prime}, 6^{\prime \prime}\right.$ trimethylcyclohex $1^{\prime \prime}$ en $\left.1^{\prime \prime} y l\right)$ ) $2(5 H)$ furanone] is structurally similar to strigolactones (Fig. 1A), including the number of $\mathrm{C}$ atoms and the pres ence of a lactone. We have named compound $\mathrm{V}$ carlactone (Fig. 1A), reflecting its origin from $\beta$ carotene and its relationship to strigolactones.

Carlactone was also produced when $9 \mathrm{cis} \beta$ apo $10^{\prime}$ carotenal was incubated with purified PsCCD8, instead of lysates of overexpressing $E$. coli cells, and in the absence of any added co factor (fig. S16). Thus, the $\mathrm{C}$ skeleton of carlactone derives entirely from 9 cis $\beta$ apo $10^{\prime}$ carotenal, and CCD8 alone is capable of catalyzing all the reactions needed to form carlactone from this substrate, including the introduction of three oxygens and intramolecular rearrangement. A hypothetical mechanism for the conversion of 9 cis $\beta$ apo $10^{\prime}$ carotenal into carlactone is presented in fig. S17.

We first performed each assay with each en zyme individually. Strigolactone biosynthesis was initiated by the D27 mediated isomeriza tion of all trans to $9 \mathrm{cis} \beta$ carotene, followed by the CCD7 catalyzed formation of 9 cis $\beta$ apo $10^{\prime}$ carotenal that was converted by CCD8 into carlactone (Fig. 1A). To investigate the ef fect of interactions between enzymes, we then combined PsCCD7 and PsCCD8 in vitro. No products were detected with all trans $\beta$ carotene as a substrate, but 9 cis $\beta$ carotene was converted into carlactone (fig. S18A). Addition of the isom erase D27 to PsCCD7 and PsCCD8 allowed the formation of carlactone from all trans $\beta$ carotene (fig. S18B).

The absence of carlactone in $d 27, c c d 7$, and $c c d 8$ mutants is the reason for the more branching, high tillering phenotype observed. We applied carlactone produced in vitro to the rice $d 27$, $\operatorname{ccd} 7$ (htd I), and $c c d 8(d 10)$ mutants and, as a control, to the wild type and to $d 3$, a high tillering strigolactone signal perception mutant (17). Af ter treatment for 28 days, tiller numbers of $d 27$, htd 1 , and $d 10$ mutants were reduced $(d 27$ from 5.3 to 3 ; htd 1 from 6 to 2.3 ; $d 10$ from 5.7 to $1.3)$, whereas wild type and $d 3$ tiller numbers were not affected (wild type, 2; $d 3,4.5$ ) (Fig. 3, $\mathrm{A}$ and $\mathrm{B})$. The activity of carlactone in restoring the wild type tillering phenotype in $d 27, \operatorname{ccd} 7$, and $c c d 8$ mutants indicates that this compound is a likely precursor of strigolactones. Only a few reactions dioxygenation followed by a de hydrogenation and simultaneous closure of the $\mathrm{B}$ and $\mathrm{C}$ rings (fig. S19B) would be required to convert carlactone into 5 deoxystrigol. This ap pears feasible in view of a recent paper that shows the chemical synthesis of the strigolactone $\mathrm{B} / \mathrm{C}$ ring system from a linear precursor in a single acid catalyzed step (29). However, we have not been able to detect carlactone in plants.

Carlactone can induce seed germination of parasitic plants. We treated Striga hermonthica seeds with various concentrations of carlactone and with GR24 as a positive control. Carlactone, although not as effective as GR24, did induce seed germination in a concentration dependent manner (Fig. 3C). The germination promoting activity of carlactone shows that the enone moiety of the $\mathrm{C}$ ring of strigolactones (Fig. 1A) is not mandatory, contradicting previous sugges tions $(30,3 I)$.

The comparatively simple structure of car lactone opens up new possibilities for designing strigolactone/carlactone analogs that could be applied to induce suicidal germination, a prom ising tool to combat root parasitic weeds that rep resent severe agricultural pests worldwide (32). Knowledge of the carlactone structure will great ly assist in identifying the bioactive compound(s) that regulate shoot branching and in elucidating further steps in strigolactone biosynthesis, in cluding the activities of $\operatorname{MAX} 1(8,14$ 17).

References and Notes

1. S. H. Schwartz, B. C. Tan, D. A. Gage, ]. A. Zeevaart,

D. R. McCarty, Science 276, 1872 (1997).

2. J. von Lintig, Annu. Rev. Nutr. 30, 35 (2010).

3. H. R. Medina, E. Cerdá Olmedo, S. Al Babili, Mol. Microbiol. 82, 199 (2011).

4. F. Bouvier, J. C. Isner, O. Dogbo, B. Camara, Trends Plant Sai. 10, 187 (2005).

5. A. R. Moise, J. von Lintig, K. Palczewski, Trends Plant Sci. 10, 178 (2005).

6. M. E. Auldridge, D. R. McCarty, H. J. Klee, Curr. Opin. Plant Biol. 9, 315 (2006).

7. M. H. Walter, D. Strack, Nat. Prod. Rep. 28, 663 (2011).

8. X. Xie, K. Yoneyama, K. Yoneyama, Annu. Rev. Phytopathol. 48, 93 (2010).

9. R. Matusova et al., Plant Physiol. 139, 920 (2005).

10. C. E. Cook, L. P. Whichard, B. Turner, M. E. Wall, G. H. Egley, Science 154, 1189 (1966).

11. K. Akiyama, K. Matsuzaki, H. Hayashi, Nature 435, 824 (2005).

12. M. Umehara et al., Nature 455, 195 (2008).

13. V. Gomez Roldan et al., Nature 455, 189 (2008).

14. O. Leyser, Dev. Cell 15, 337 (2008).

15. O. Leyser, Plant Cell Environ. 32, 694 (2009).

16. C. A. Beveridge, ]. Kyozuka, Curr. Opin. Plant Biol. 13 34 (2010).

17. Y. Wang, J. Li, Curr. Opin. Plant Biol. 14, 94 (2011).

18. S. H. Schwartz, X. Qin, M. C. Loewen, J. Biol. Chem. 279, 46940 (2004).

19. M. Schlicht et al., Plant ]. 55, 709 (2008).

20. A. Alder, I. Holdermann, P. Beyer, S. Al Babili, Biochem. J. 416, 289 (2008).

21. S. H. Schwartz, X. Qin, J. A. D. Zeevaart, J. Biol. Chem. 276, 25208 (2001).

22. D. DellaPenna, B. J. Pogson, Annu. Rev. Plant Biol. 57, 711 (2006).

23. Q. Yu, S. Ghisla, ]. Hirschberg, V. Mann, P. Beyer, J. Biol. Chem. 286, 8666 (2011).

24. G. E. Bartley, P. A. Scolnik, P. Beyer, Eur. J. Biochem. 259, 396 (1999).

25. L. Zechmeister, Cis trans Isomeric Carotenoids, Vitamin $A$ and Arylpolyenes (Springer Verlag, Heidelberg, 1962), chap. V.

26. H. Lin et al., Plant Cell 21, 1512 (2009),

27. G. Englert, in Carotenoids, Vol. 1B: Spectroscopy, G. Britton, S. Liaaen Jensen, H. Pfander, Eds. (Birkhäuser, Basel, 1995), chap. 6.

28. ACD Shift Prediction Software, Version 11.02, Advanced Chemistry Development Inc. (2008). 
29. K. Chojnacka et al., Org. Biomol. Chem. 9, 5350 (2011).

30. K. Yoneyama, X. Xie, K. Yoneyama, Y. Takeuchi, Pest Manag. Sci. 65, 467 (2009).

31. B. Zwanenburg, A. S. Mwakaboko, A. Reizelman, G. Anilkumar, D. Sethumadhavan, Pest Manag. Sci. 65, 478 (2009).

32. C. Parker, Pest Manag. Sci. 65, 453 (2009).

Acknowledgments: We thank S. Schürch and C. Bühr for

technical help with ESI MS analysis, E. Decker for valuable discussions, B. Zwanenburg for supplying GR24, W. Boland for $\beta$ apo 13 carotenone, Y. Wang for seeds of rice $d 27$, L. Zhu for seeds of htd 1, and J. Kyozuka for seeds of $d 3$ and $d 10$ mutants. Supported by the Deutsche Forschungsgemeinschaft (grant AL892/1 4 and the Graduiertenkolleg 1305 "Plant Signal Systems"), the Higher Education Commission of Pakistan, the Netherlands Organization for Scientific Research (VICl grant

865.06.002 and equipment grant 834.08.001), and the Centre for BioSystems Genomics, a part of the Netherlands Genomics Initiative/Netherlands Organization for Scientific Research. Materials are available under an MTA agreement with the University of Freiburg. The University of Freiburg has filed
European patent application (number 11003 470.9) on carlactone and its applications. 